Ultra-fast Laser Synthesis of Nanopore Arrays in Silicon for Bio-molecule Separation and Detection

J. W. Tringe, N. lleri, S. E. Letant, P. Stroeve, M. Shirk, S. Zaidi, R. L. Balhorn, C. W. Siders

February 11, 2008 
This document was prepared as an account of work sponsored by an agency of the United States government. Neither the United States government nor Lawrence Livermore National Security, LLC, nor any of their employees makes any warranty, expressed or implied, or assumes any legal liability or responsibility for the accuracy, completeness, or usefulness of any information, apparatus, product, or process disclosed, or represents that its use would not infringe privately owned rights. Reference herein to any specific commercial product, process, or service by trade name, trademark, manufacturer, or otherwise does not necessarily constitute or imply its endorsement, recommendation, or favoring by the United States government or Lawrence Livermore National Security, LLC. The views and opinions of authors expressed herein do not necessarily state or reflect those of the United States government or Lawrence Livermore National Security, LLC, and shall not be used for advertising or product endorsement purposes.

This work performed under the auspices of the U.S. Department of Energy by Lawrence Livermore National Laboratory under Contract DE-AC52-07NA27344. 


\title{
Ultra-fast Laser Synthesis of Nanopore Arrays in Silicon for Bio-molecule Separation and Detection
}

\author{
J. Tringe ${ }^{1}$, N. Ileri ${ }^{2}$, S. Létant ${ }^{1}$, P. Stroeve ${ }^{2}$, M. Shirk ${ }^{1}$, S. Zaidi ${ }^{3}$, R. Balhorn ${ }^{1}$ and C. Siders ${ }^{1}$ \\ ${ }^{1}$ LLNL ${ }^{2}$ UC Davis ${ }^{3}$ Gratings, Inc.
}

\begin{abstract}
We demonstrate that interference of ultra-fast pulses of laser light can create regular patterns in thin silicon membranes that are compatible with the formation of a uniform array of nanopores. The spacing and size of these pores can be tuned by changing the laser energy, wavelength and number of ultra-short pulses. Short pulses and wavelengths ( $~ 550 \mathrm{~nm}$ and smaller) are needed to define controllable nanoscale features in silicon. Energy must be localized in time and space to produce the etching, ablation or amorphization effects over the $\sim 100 \mathrm{~nm}$ length scales appropriate for definition of single pores. Although in this brief study pattern uniformity was limited by laser beam quality, a complementary demonstration reported here used continuous-wave interferometric laser exposure of photoresist to show the promise of the ultra-fast approach for producing uniform pore arrays. The diameters of these interferometrically-defined features are significantly more uniform than the diameters of pores in state-of-the-art polycarbonate track etch membranes widely used for molecular separations.
\end{abstract}

\section{Motivation}

For protein screening, organic and inorganic molecular development, and also for pre-screening of toxins and other molecules for sensor applications, there is a need for filters with uniform pore size, scalable between 1-100 nm. Advances in the development of silicon and other materials with nanometer-scale (1$1000 \mathrm{~nm}$ ) pores[1, 2] or slits raise the possibility of producing sizing filters with a sufficiently large dynamic range of size selection (extending from $\sim 1 \mathrm{~nm}$ to $1 \mu \mathrm{m}$ ) to cover, for example, the entire range of known sizes of proteins and protein complexes. However, the smallest of these feature sizes are beyond the reach of standard lithographic processes for large areas (1-100's of $\mathrm{mm}^{2}$ ) required for most applications.

Non-lithographic methods have been developed for producing near-nanometer pore sizes: for example, porous membranes have been created through anodic etching of $\mathrm{Al}[3,4]$, and mesoporous silica formed through sol-gel process[5]. Although these methods have been moderately successful for certain applications, their usefulness is inherently limited due to lack of uniformity or scalability. For example, pore dimensions typically vary over a broad range: $~ 30-400 \mathrm{~nm}$ for anodic alumina and $\sim 2-20 \mathrm{~nm}$ in solgel films. Other filter materials such as zeolites have ultra-uniform pores, but only in the relatively narrow range of $\sim 0.3-3 \mathrm{~nm}$.[6] Carbon nanotubes are being developed at LLNL for filters in the 1-10 nm range, but scaling beyond this limit is extremely challenging, and the cylindrical shape of the pores may present additional complications. Finally, ion-track etching through polycarbonate films can produce a wide range ( $\sim 10 \mathrm{~nm}$ to $\sim \mu \mathrm{m})$ of pore diameters, but uniformity and flow rates are limited to $\pm 20 \%$ and $<0.1$ $\mathrm{mL} / \mathrm{min} / \mathrm{cm}^{2}$ (for $10 \mathrm{~nm}$ diameter pores), respectively.[7]

Here we describe preliminary results which demonstrate the promise of new method to fabricate ultrasmall, uniform filters in silicon by interferometric pulsed laser exposure (IPLE). This approach eliminates errors in critical dimensions associated with imaging of organic photoresist layers by directly inducing local phase changes in silicon. Nanoscale features are defined through interference of two or more laser beams incident simultaneously on the same surface. Three-dimensional features in silicon are formed at high laser energies by direct ablation, or at lower energies by inducing less disruptive phase changes such as localized crystallization or amorphization. For these latter phase changes, etching processes can subsequently be employed to create three-dimensional structures in silicon since different phases (e.g., amorphous vs. crystalline) of silicon etch at different rates. 


\section{Approach}

To understand our proposed approach, consider first the standard operation mode of interferometric laser exposure (ILE) in photoresist. Two or more coherent laser beams are simultaneously incident on a surface coated with a thin film of photoresist, such that the intensity at the surface is modulated by the interference pattern. Photoresist is exposed in domains of constructive interference. For example, parallel lines with widths as small as $135 \mathrm{~nm}$ can be easily created by applying two 257$\mathrm{nm}$ laser beams incident at 80 degrees angular separation on a planar substrate, as

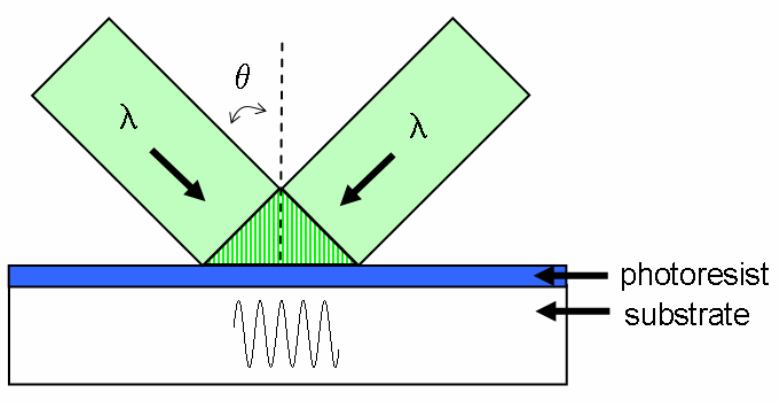

$$
\text { period }=\lambda / 2 \sin (\theta)
$$

Figure 1. Interferometric laser exposure of photoresist shown in Fig. 1. Patterns in the exposed, developed photoresist can then be transferred to silicon by etching. A potential limitation of this method is that feature size is a sensitive function of photoresist thickness and resolution; both can be variable over relatively small areas. For direct patterning in $\mathrm{Si}$, however, photoresist variability is not an issue. Small features can be created since the absorption length of light in $\mathrm{Si}$ is $\sim 10 \mathrm{~nm}$ for wavelengths below $400 \mathrm{~nm}$.

To create pores, a pulse of interferometrically-defined laser light is directed to the surface of the silicon-oninsulator wafer. Multiple coherent beams simultaneously interfere to create an array of spots, as shown in Fig. 2(a). At high laser intensities, the silicon is simply vaporized (ablated) locally, leaving holes in the top layer of silicon, as shown in Fig. 2(b).[8] It is important to note that the pore is defined by the intersection of the parabolic ablated volume with the planar interface between the silicon-on-insulator and buried oxide. For this reason, the pore diameter can be much smaller than the diameter of the ablated domain at the top silicon surface. We estimate that pores as small as $\sim 10 \mathrm{~nm}$ can be created with this approach, using ablated volumes with top diameters larger than $100 \mathrm{~nm}$.

\section{(a)}

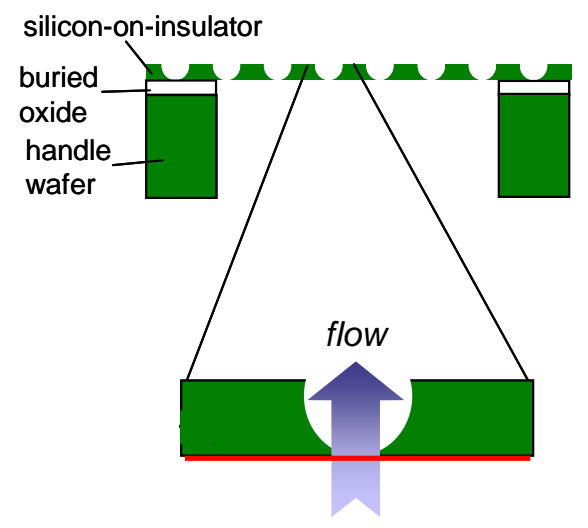

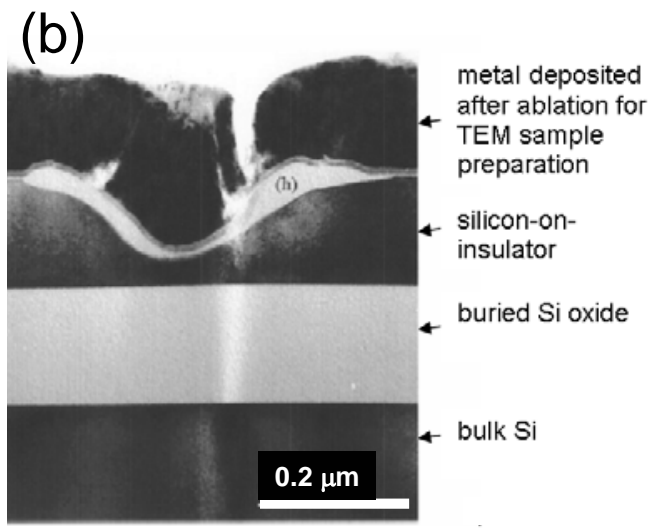

Figure 2. (a) Features created by interferometric pulsed laser exposure of a silicon-on-insulator structure. (b) Cross-sectional TEM of a hole formed in silicon by focused (not interferometrically-defined) laser beam: 5 pulses of $150 \mathrm{ps}$ of 258-nm light from a Ti-Sapphire laser, $475 \mathrm{~nm}$ beam diameter. ${ }^{12}$

Under different irradiation conditions, silicon can be locally melted, as opposed to being ablated. The melting rate and extent, measured by reflectivity, can be precisely controlled with the time and energy of the laser pulse.[9] Melted Si can remain amorphous upon cooling, or amorphous silicon can be recrystallized, depending on laser energy, wavelength and pulse duration.[10] Laser-amorphized Si can be 
selectively removed from the crystalline matrix because amorphous silicon etches 500 times faster than crystalline silicon in hydrogen plasma.[11]

For pore synthesis, critical issues are thermal energy transport and atomic mobility at interfaces at very short times in the Si crystal lattice, since these define the size and shape of the pore. A landmark paper by Liu et al. demonstrated that for a single pulse of 20 ps $532 \mathrm{~nm}$ laser energy incident on a (111) crystalline Si surface, there exists a critical threshold energy density of $0.2 \mathrm{~J} / \mathrm{cm}^{2}$, necessary to induce an amorphous phase transition.[12] Time-resolved reflectivity and charge emission data, as well as a unique ring shape generated by the Gaussian laser spot size on the surface, were all explained in terms of rapid $\left(10^{-11} \mathrm{~s}\right)$ lattice thermalization. This model contrasted with non-thermal melting modes which have been observed, for example, by time-resolved x-ray diffraction in Ge.[13] However, the amorphous regions created in Si were tens of microns across, and no attempt was made to examine the three-dimensional shape of the transformed volume. More recent studies have demonstrated that much smaller volumes can be amorphized, and even ablated, with a tightly focused beam.[8] Other two-dimensional surface experiments indicate that the transformation rate depends on Si crystallographic direction,[14] and on laser wavelength and pulse duration.[10]

\section{Demonstration of interference effects with ultra-fast laser pulses}

We were successful in demonstrating the proposed effect, in which a solid crystalline surface can be modified according to the optical intensity pattern defined by interference. For these experiments, we employed a simple optical design, as shown in Fig. 3. Laser light is expanded and collimated, then passed through a converging lens in which the central part of the beam is blocked by a structure which is as wide in one dimension as the diameter of the lens. The remaining fraction of the light is focused to the target, where the two parts of the beam on either side of the block recombine and interfere.

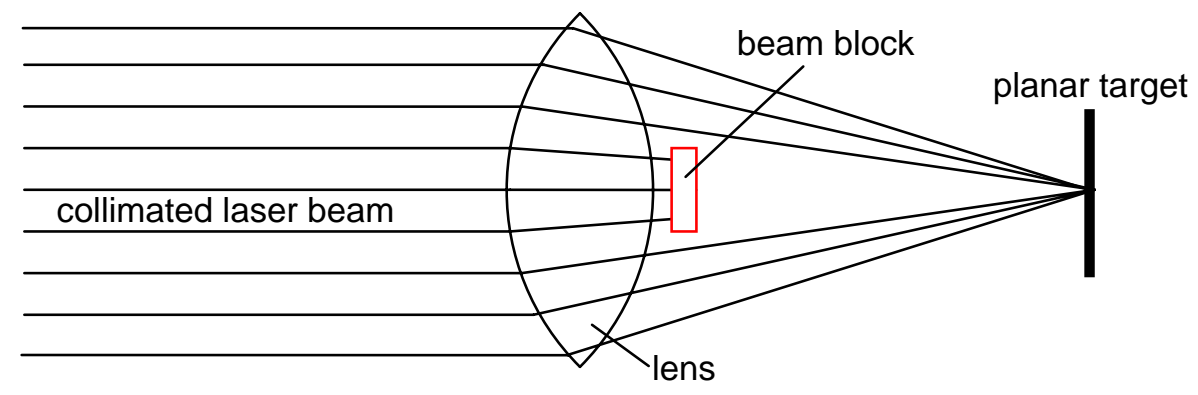

Figure 3. Lens and target configuration for achieving interferometrically-defined ablation

The scanning electron microscope image Fig. 4(a) shows results obtained using this method in thin silicon in a silicon-on-insulator configuration. Lines separated by about $2 \mu \mathrm{m}$ were created, extending many tens of microns up and down beyond the field of view. (The square darker contrast in the center of Fig. 4(a) is an artifact related to the scanning electron microscope.) Here direct ablation was achieved in air with ultra-short pulses of $533 \mathrm{~nm}$ light. It is useful to note that the size of the ablated feature can be controlled with the wavelength of the light, since the absorption depth in silicon is a strong function of wavelength. Some imperfections are apparent in the lines in $\mathrm{Si}$, which are due to non-uniformities in the laser beam used for these experiments.

Fig. 4(b) shows a similar interference pattern created with the same laser beam, but on a diamond substrate in air. Uniformity is improved in this case, likely because carbon reacts with oxygen in air during the ablation process, producing a smoother mass transfer process from solid to vapor phase. A similar process could be achieved in silicon if the wafer could be exposed in a reactive ambient such as $\mathrm{SF}_{6}$. Alternately, the silicon could be simply amorphized at lower beam energies. 

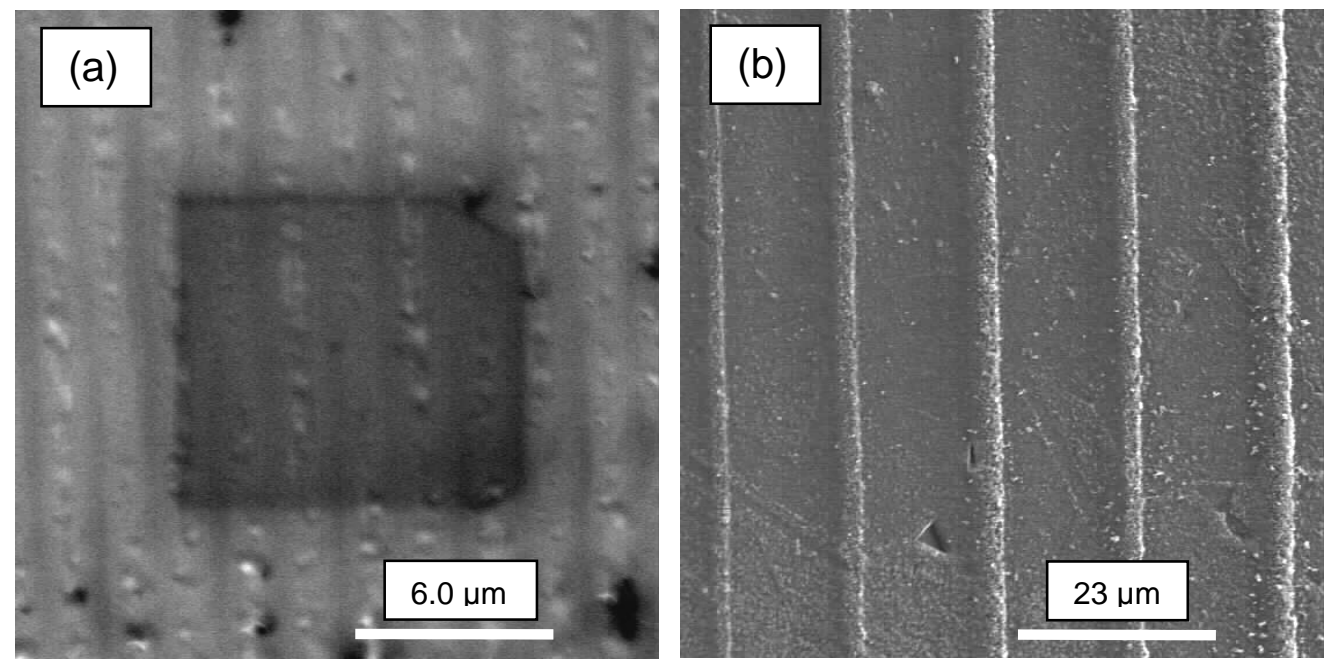

Figure 4. 1D patterns created with 533-nm wavelength two-beam interference of ultra-short pulses in air: (a) silicon substrate in SOI configuration (b) diamond substrate with etching in reactive oxygen ambient

One-dimensional patterns as shown in Fig. 4 are created when interfering beams are at a constant angle with respect to one another over the surface of the target. If higher, non-planar optical modes are present in the interfering laser beam profile, multiple-period two-dimensional patterns, typically referred to as Moirépatterns, are created as shown in Fig. 5(a). This pattern was formed in amorphous silicon-on-silicon in air with five pulses of $533 \mathrm{~nm}$ laser light. Fig. 5(b) shows the result for the same exposure conditions with 10 pulses. Again, non-uniformities arise from the suboptimal beam quality, but the principle is shown in which two-dimensional patterns can be readily achieved with this fast, simple process.
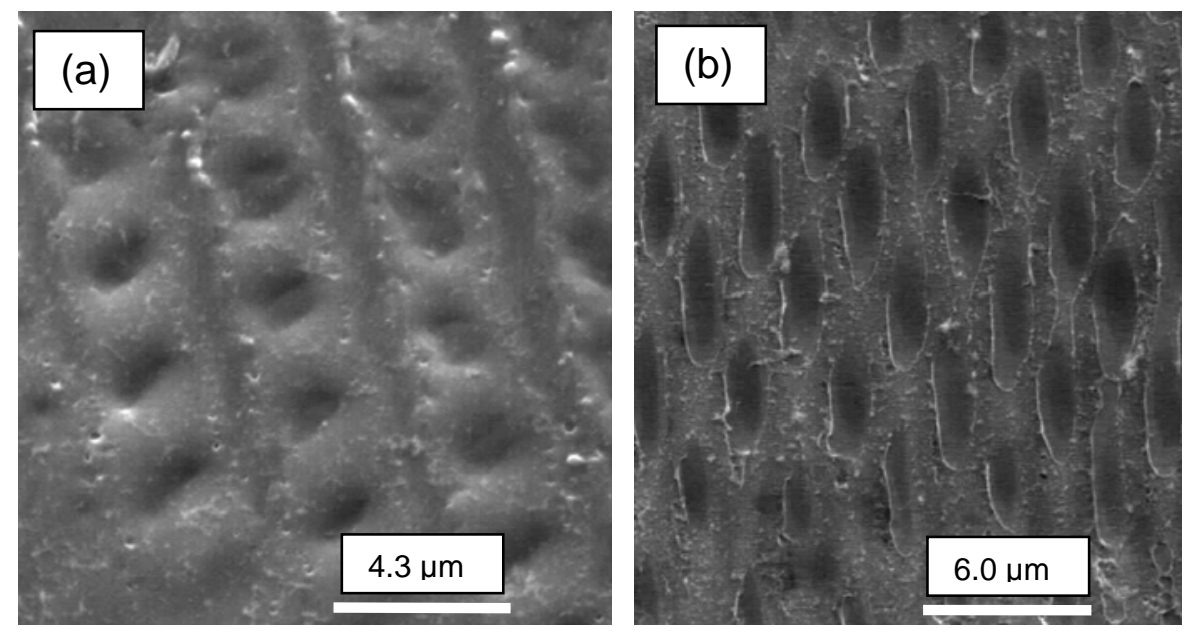

Figure 5. 2D patterns created with 533-nm wavelength two-beam interference of ultra-short pulses in air for amorphous silicon on a crystalline silicon substrate, (a) five pulses (b) ten pulses

\section{Improving pattern uniformity}

In these experiments uniformity of ultra-fast patterns was limited by the quality of the available beam. This in no way represents a fundamental limitation for the proposed process, however. Our beams were far from ideal, and we had limited time and resources for optimization. To better demonstrate the full potential of interferometric pattern definition, we therefore exposed photoresist on silicon wafers using a continuouswave laser. Although the resolution of this approach will ultimately be limited by the properties of the photoresist itself, nevertheless this process could be very useful for a range of applications such as separations of viruses where individual particles are closer to $100 \mathrm{~nm}$ or larger. 
Fig. 6(a) is a top-down scanning electron micrograph showing the uniformity of a pattern defined in photoresist that is achieved by interferometric lithography. The inset is a cross-sectional view indicating the shape of the photoresist pillars. To create this pattern, two sequential exposures were made of the same photoresist layer, when the substrate was rotated by $90^{\circ}$ between exposures.

For comparison, a commercially-available polycarbonate track-etched (PCTE) membrane is shown in Fig.6(b). This polycarbonate filter, about $5 \mu \mathrm{m}$ thick, contains many cylindrical holes which were defined by the damage paths of energetic ions that passed completely through the membrane. These damaged regions were converted to holes by a subsequent etching process which selectively removed the polycarbonate around the ion tracks.
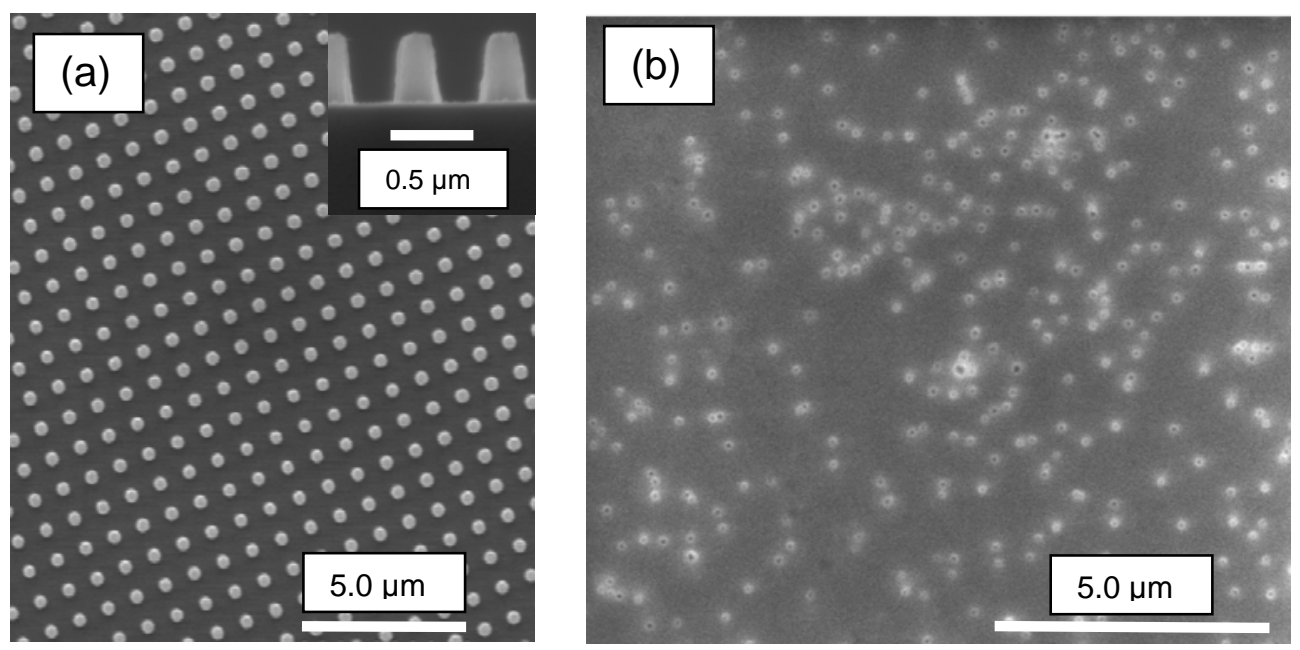

Figure 6. Scanning electron microscope image of (a) photoresist defined by interferometric lithography, and (b) polycarbonate track-etched (PCTE) membrane

The uniformity of the patterns is quantified in Table I, where the standard error (average divided by standard deviation) of the diameters is significantly improved with the interferometrically-defined photoresist: 0.76 for PCTE vs. 0.15 for interferometric lithography. Additional processing steps are necessary to create a full porous membrane from the photoresist pattern, but these results are promising for both the photoresist approach as well as for ultra-fast pattern definition.

Table I: Feature uniformity in polycarbonate-track etched membrane and photoresist on $\mathrm{Si}$

\begin{tabular}{|c|c|c|c|}
\hline & $\begin{array}{l}\text { Diameter } \\
(\mathrm{nm})\end{array}$ & $\begin{array}{l}\text { Std Dev. } \\
(\mathrm{nm})\end{array}$ & Std Error \\
\hline PCTE membrane & 326 & 249 & 0.76 \\
\hline Photoresist on Si & 270 & 41 & 0.15 \\
\hline
\end{tabular}

\section{Future steps}

Preliminary results from this LDRD feasibility study have been complemented by new Si membrane results obtained with support from a 2-year, \$50k/year GREAT (Graduate Research and Education in Adaptive bio-Technology) grant from the State of California. The GREAT program has funded a UC Davis graduate student, Nazar Ileri, since July of 2007. Ms. Ileri is presently synthesizing Si nanofilter membranes using unique interferometric lithography resources at LLNL, in partnership with UC Davis and a commercial partner, Gratings, Inc. We have achieved encouraging results with our preliminary structures, and plan to fabricate our first porous membranes in February/March 2008. An abstract describing our results has been accepted for oral presentation at the Spring 2008 Materials Research Society Meeting in San Francisco. We are now initiating a new collaboration to help us efficiently address the beam quality issues that limited 
pattern uniformity in this LDRD effort, and to demonstrate the full potential of this direct-write technique for fast, simple fabrication of large arrays of identical nanometer-scale pores. We will be submitting proposals to NIH, DARPA and DHS to develop this technology and apply it to rapid separation and characterization of proteins, viruses and toxins.

\section{Summary}

We successfully demonstrated the principle requirements for direct-write ultra-fast laser pore synthesis in thin silicon films: regular patterns can be readily created using interferometrically-defined laser light, and these patterns can be tuned with laser wavelength and pulse duration. Uniformity in the structures produced by ultra-short pulse laser processing in this study was limited by laser beam quality, but this does not represent a fundamental obstacle. In fact, continuous-wave laser exposure of photoresist indicates that a very high degree of uniformity can be achieved with the interferometric approach, significantly better than the pore size uniformity in state-of-the-art polycarbonate track-etched membranes. We are presently using knowledge developed in this study to synthesize our first nanoporous membranes.

\section{Acknowledgements}

We are grateful to Dr. Jerry Britten, Dr. Hoang Nguyen and Cindy Larson for assistance with continuouswave exposure of photoresist on silicon, and to Dr. Brent Stuart and Dr. Jerry Benterou for guidance on ultra-fast laser beam shaping. We also gratefully acknowledge Prof. Roland Faller and Professor Ahmet Palazoglu (UC Davis), and the University of California Biotechnology program GREAT, for advisory and financial support, respectively, of NI.

\section{References}

[1] T. Tada, A. Hamoudi, T. Kanayama, and K. Koga, Appl. Phys. Lett., vol. 70, pp. 2358, 1997.

[2] V. Lehman and H. Foll, J. Electrochem. Soc., vol. 137, pp. 990.

[3] A. P. Li, F. Muller, A. Birner, K. Nielsch, and U. Gosele, J. Appl. Phys., vol. 84, pp. 6023, 1998.

[4] H. Masuda and K. Fukuda, Science, vol. 268, pp. 1466, 1995.

[5] W. Chen, W. Cai, L. Zhang, G. Wang, and L. Zhang, Journal of Colloid and Interface Science, vol. 238, pp. 291, 2001.

[6] I. Lenntech, "Regenerative adsorption zeolite," 2006.

[7] Sterilitech, "Sterlitech Polycarbonate Track Etch (PCTE) Membranes," 2006.

[8] J. Jia, M. Li, and C. V. Thompson, "Amorphization of silicon by femtosecond laser pulses," Applied Physics Letters, vol. 84, pp. 3205-3207, 2004.

[9] M. Hernandez, J. Venturini, D. Zahorski, J. Boulmer, D. Debarre, G. Kerrien, T. Sarnet, C. Laviron, M. N. Semeria, D. Camel, and J. L. Santailler, "Laser thermal processing for ultra shallow junction formation: numerical simulation and comparison with experiments," Applied Surface Science, vol. 208, pp. 345-351, 2003.

[10] S. Higashi and T. Sameshima, "Pulsed-laser-induced microcrystallization and amorphization of silicon thin films," Japanese Journal of Applied Physics Part 1-Regular Papers Short Notes \& Review Papers, vol. 40, pp. 480-485, 2001.

[11] R. N. Jacobs, A. J. Stoltz, E. W. Robinson, P. R. Boyd, L. A. Almeida, J. H. Dinan, and L. Salamanca-Riba, "Processing and characterization of a-Si : H photoresists for a vacuum-compatible photolithography process," Journal of Electronic Materials, vol. 33, pp. 538-542, 2004.

[12] J. M. Liu, R. Yen, H. Kurz, and N. Bloembergen, "Phase-Transformation on and Charged-Particle Emission from a Silicon Crystal-Surface, Induced by Picosecond Laser-Pulses," Applied Physics Letters, vol. 39, pp. 755-757, 1981.

[13] C. W. Siders, A. Cavalleri, K. Sokolowski-Tinten, C. Toth, T. Guo, M. Kammler, M. H. von Hoegen, K. R. Wilson, D. von der Linde, and C. P. J. Barty, "Detection of nonthermal melting by ultrafast X-ray diffraction," Science, vol. 286, pp. 1340-1342, 1999.

[14] J. A. Yater and M. O. Thompson, "Orientation Dependence of Laser Amorphization of Crystal Si," Physical Review Letters, vol. 63, pp. 2088-2091, 1989. 


\section{Disclaimer}

This document was prepared as an account of work sponsored by an agency of the United States government. Neither the United States government nor Lawrence Livermore National Security, LLC, nor any of their employees makes any warranty, expressed or implied, or assumes any legal liability or responsibility for the accuracy, completeness, or usefulness of any information, apparatus, product, or process disclosed, or represents that its use would not infringe privately owned rights. Reference herein to any specific commercial product, process, or service by trade name, trademark, manufacturer, or otherwise does not necessarily constitute or imply its endorsement, recommendation, or favoring by the United States government or Lawrence Livermore National Security, LLC. The views and opinions of authors expressed herein do not necessarily state or reflect those of the United States government or Lawrence Livermore National Security, LLC, and shall not be used for advertising or product endorsement purposes.

\section{Auspices Statement}

This work performed under the auspices of the U.S. Department of Energy by Lawrence Livermore National Laboratory under Contract DE-AC52-07NA27344.

This work was funded by the Laboratory Directed Research and Development Program at LLNL under project tracking code 07-FS-001. 